Pacific Journal of Mathematic 


\title{
CONGRUENCES ON REGULAR SEMIGROUPS
}

\author{
N. R. REILly AND H. E. Scheiblich
}

For any regular semigroup $S$ the relation $\theta$ is defined on the lattice, $\Lambda(S)$, of congruences on $S$ by: $(\rho, \tau) \in \theta$ if and only if $\rho$ and $\tau$ induce the same partition of the idempotents of $S$. Then $\theta$ is an equivalence relation on $\Lambda(S)$ such that each equivalence class is a complete modular sublattice of $\Lambda(S)$. If $S$ is an inverse semigroup then $\theta$ is a congruence on $A(S)$, $\Lambda(S) / \theta$ is complete and the natural homomorphism of $\Lambda(S)$ onto $\Lambda(S) / \theta$ is a complete lattice homomorphism.

Any congruence on an inverse semigroup $S$ can be characterized in terms of its kernel, namely, the set of congruence classes containing the idempotents of $S$. In particular, any congruence on $S$ induces a partition of the set $E_{S}$ of idempotents of $S$ satisfying certain normality conditions. In this note, those partitions of $E_{S}$ which are induced by congruences on $S$ and the largest and smallest congruences on $S$ correspond ing so such a partition of $E_{S}$ are characterized.

1. Preliminary results and definitions. We adopt the notation and terminology of Clifford and Preston [2]. A semigroup $S$ is called regular if $a \in a S a$, for all $a \in S$. If, for all $a \in S$, there exists an element $b \in S$ such that $a b a=a$ and $b a b=b$ then we say that $b$ is an inverse of $a$ and that $(a, b)$ is a regular pair [11]. In a regular semigroup, every element has an inverse. An inverse semigroup is a semigroup in which each element has a unique inverse. The elementary properties of regular and inverse semigroups can be found in [2]. In particular, a semigroup $S$ is an inverge semigroup if and only if it is regular and its idempotents commute ([2], Th. 1.17). The inverse of an element $a$ is then denoted by $a^{-1}$. For any idempotent $e, e^{-1}=e$, and, for any elements $a, b$ of $S$

$$
\left(a^{-1}\right)^{-1}=a,(a b)^{-1}=b^{-1} a^{-1} .
$$

If $\left(a, a^{\prime}\right)$ is a regular pair, then $a a^{\prime}$ and $a^{\prime} a$ are both idempotents but are not always equal (even in an inverse semigroup).

A regular (inverse) subsemigroup $T$ of a semigroup $S$ is just a subsemigroup of $S$ which is a regular (inverse) semigroup in its own right.

For any semigroup $S$ we shall denote by $E_{S}$ the set of idempotents of $S$. The set $E_{S}$ can be partially ordered by defining $e \leqq f$ if and only if $e f=f e=e$. Of course, if $S$ is an inverse semigroup then this reduces to $e f=e$ and $E_{S}$ then becomes a semilattice, with $e \wedge f=$ ef, called the semilattice of idempotents of $S$. 
We shall call a subset $A$ of a partially ordered set $B$ convex if $x \leqq y \leqq z, x, z \in A$ implies that $y \in A$.

If $\rho$ is a congruence on a semigroup $S$ then we shall denote by $\left.\rho\right|_{E_{s}}$ the partition of $E_{S}$ induced by $\rho$, that is $\left.\rho\right|_{E_{s}}=\rho \cap\left(E_{S} \times E_{S}\right)$, and by $a \rho$ the $\rho$-class containing the element $a$. We shall also make use of the fact that, in an inverse semigroup, if $(a, b) \in \rho$ then $\left(a^{-1}, b^{-1}\right) \in \rho$ ([4] Corollary 2.3) and, consequently, $\left(a a^{-1}, b b^{-1}\right) \in \rho$.

Clearly a homomorphic image of a regular semigroup is regular and it was established in [12] that a homomorphic image of an inverse semigroup is an inverse semigroup.

Finally, two elements of a semigroup $S$ are said to be $\mathscr{L}-(\mathscr{R}-)$ equivalent if they generate the same principal left (right) ideal of $S$. Clearly $\mathscr{L}$ and $\mathscr{R}$ are equivalence relations on $S$, as is the relation $\mathscr{H}$, defined by $\mathscr{H}=\mathscr{L} \cap \mathscr{R}$.

2. Maximal regular subsemigroups. In this section we shall generalize the well known result that for any idempotent $e$ of a semi-group $S$ there is a unique maximum subgroup of $S$ with identity $e$.

Lemma 1.1. Let $\left(a, a^{\prime}\right)$ and $\left(b, b^{\prime}\right)$ be regular pairs in a semigroup $S$. Then $a^{\prime} a b b^{\prime}$ and $b b^{\prime} a^{\prime} a$ are idempotents of $S$ if and only if ( $a b$, $\left.b^{\prime} a^{\prime}\right)$ is a regular pair.

Proof. Let $a^{\prime} a b b^{\prime}$ and $b b^{\prime} a^{\prime} a$ be idempotents. Then

$$
(a b)\left(b^{\prime} a^{\prime}\right)(a b)=a a^{\prime} a b b^{\prime} a^{\prime} a b b^{\prime} b=a a^{\prime} a b b^{\prime} b=a b
$$

and

$$
\left(b^{\prime} a^{\prime}\right)(a b)\left(b^{\prime} a^{\prime}\right)=b^{\prime} b b^{\prime} a^{\prime} a b b^{\prime} a^{\prime} a a^{\prime}=b^{\prime} b b^{\prime} a^{\prime} a a^{\prime}=b^{\prime} a^{\prime}
$$

as required.

Conversely, if $\left(a b, b^{\prime} a^{\prime}\right)$ is a regular pair, then

$$
\left(a^{\prime} a b b^{\prime}\right)\left(a^{\prime} a b b^{\prime}\right)=a^{\prime}(a b)\left(b^{\prime} a^{\prime}\right)(a b) b^{\prime}=a^{\prime} a b b^{\prime}
$$

and similarly $b b^{\prime} a^{\prime} a$ is an idempotent.

LeMma 1.2. ([5] Lemma 1.1) If $e$ and $f$ are idempotents in a regular semigroup $S$ then, for some idempotent $g$ in $S,(g, e f)$ is a regular pair.

Lemma 1.3. For a regular semigroup $S$ the following are equivalent:

(1) $E_{S} E_{S} \subseteq E_{s}$

(2) $e \in E_{S},(e, x)$ a regular pair implies that $x \in E_{S}$; 
(3) $\left(a, a^{\prime}\right)$ and $\left(b, b^{\prime}\right)$ regular pairs implies that $\left(a b, b^{\prime} a^{\prime}\right)$ is a regular pair.

Proof. (1) imples (2). (Cf. [11] Th. 1.) Let $e \in E_{S}$ and $(e, x)$ be a regular pair. Then $x=x e x=x e e x \in E_{S} E_{S} \subseteq E_{S}$. (2) implies (1). Let $e, f \in E_{s}$, then by Lemma 1.2, for some idempotent $g,(g, e f)$ is a regular pairs and so $e f \in E_{S}$.

(1) implies (3) by Lemma 1.1.

(3) implies (1). Let $e, f \in E_{s}$. Then, since $(e, e)$ and $(f, f)$ are regular pairs, $(e f, f e)$ is also a regular pair. Then $e f=(e f)(f e)(e f)=$ $(e f)(e f)$ and $e f \in E_{S}$.

Lemma 1.4. Let $S$ be a regular semigroup such that $E_{S}$ is a subsemigroup. Then, for any regular pair $\left(a, a^{\prime}\right)$

$$
a E_{S} a^{\prime} \subseteq E_{S} .
$$

Proof. Let $\left(a, \alpha^{\prime}\right)$ be a regular pair and $e \in E_{S}$. Since

$$
a^{\prime} a e \in E_{S} E_{S} \subseteq E_{S}
$$

we have

$$
\left(a e a^{\prime}\right)^{2}=a e a^{\prime} a e a^{\prime}=a a^{\prime} a e a^{\prime} a e a^{\prime}=a a^{\prime} a e a^{\prime}=a e a^{\prime} .
$$

THEOREM 1.5. Let $E$ be an idempotent subsemigroup of a semigroup $S$. Then $E^{c}=\left\{x \in S\right.$ : for some $x^{\prime},\left(x, x^{\prime}\right)$ is a regular pair, $x x^{\prime}, x^{\prime} x \in E, x E x^{\prime} \subseteq E$ and $\left.x^{\prime} E x \subseteq E\right\}$ is the largest regular subsemigroup of with $E$ as its set of idempotents.

Proof. Let $a, b \in E^{c}$ and let $a^{\prime}, b^{\prime}$ be elements of $S$ such that $a, a^{\prime}$ and $b, b^{\prime}$ satisfy the conditions of membership for $a$ and $b$, respectively. Then clearly $a^{\prime}, b^{\prime} \in E^{c}$. Moreover, since $a^{\prime} a, b b^{\prime} \in E$, we have $a^{\prime} a b b^{\prime}$ and $b b^{\prime} a^{\prime} a$ contained in $E$ and so, by Lemma 1.1, $\left(a b, b^{\prime} a^{\prime}\right)$ is a regular pair. Also

$$
a b b^{\prime} a^{\prime} \in a E a^{\prime} \subseteq E, b^{\prime} a^{\prime} a b \in b^{\prime} E b \subseteq E
$$

and

$$
a b E b^{\prime} a^{\prime} \subseteq a E a^{\prime} \leqq E, b^{\prime} a^{\prime} E a b \subseteq b^{\prime} E b \subseteq E .
$$

Thus $a b \in E^{c}$ and $E^{c}$ is a subsemigroup of $S$. Since, as pointed out above, for each $a \in E^{C}$ some inverse of $a$ is also in $E^{C}, E^{C}$ is a regular subsemigroup of $S$.

To show that $E_{E^{c}}=E$, let $a$ be an idempotent in $E^{c}$ and let $\left(a, a^{\prime}\right)$ be a regular pair with $a, a^{\prime} \in E^{c}$. Then

$$
a^{\prime}=a^{\prime} a a^{\prime}=a^{\prime} a \alpha a^{\prime} \in E E \subseteq E
$$


and so

$$
a=a a^{\prime} a=a a^{\prime} a^{\prime} a \in E E \subseteq E
$$

Hence $E_{E^{c}}=E$, since clearly $E \subseteq E_{E^{c}}$.

Now suppose that $T$ is a regular subsemigroup of $S$ such that $E_{T}=E$. Then, for any $x \in T$, there exists an $x^{\prime} \in T$ such that $\left(x, x^{\prime}\right)$ is a regular pair in $T$ and so $x x^{\prime}, x^{\prime} x \in E_{T}=E$. Then, since $E E \subseteq E$, we have, by Lemma 1.4 ,

$$
x E x^{\prime} \subseteq E \text { and } x^{\prime} E x \subseteq E
$$

Thus $x \in E^{C}$.

Corollary 1.6. Let $E$ be a subsemigroup of commuting idempotents of a semigroup $S$. Then $E^{C}$ (defined as in Theorem 1.5) is the largest inverse subsemigroup of $S$ with $E$ as its set of idempotents.

Proof. By Theorem 1.5, $E^{C}$ is the largest regular subsemigroup with $E$ as its set of idempotents. Since the elements of $E$ commute, $E^{C}$ is, in fact, an inverse subsemigroup and so is clearly the largest such.

3. The lattice of congruences on a regular semigroup. For any semigroup $S$ we denote by $\Lambda(S)$ the lattice of congruences on $S$.

If $\sigma$ and $\rho$ are congruences on a semigroup $S$ such that $\sigma \subseteq \rho$ then the relation $\rho / \sigma$ on $S / \sigma$ defined by

$$
\rho / \sigma=\{(x \sigma, y \sigma) \in S / \sigma \times S / \sigma:(x, y) \in \rho\}
$$

is a congruence on $S / \sigma$. Moreover, the mapping $\rho \rightarrow \rho / \sigma$ is a one-toone order preserving mapping of the congruences $\rho$ on $S$ containing $\sigma$ onto the congruences on $S / \sigma$, as is easy to show.

It is straight forward to verify that if $\sigma, \rho, \tau \in \Lambda(S)$ and $\sigma \subseteq \rho, \tau$ then

$$
(\rho \cap \tau) / \sigma=\rho / \sigma \cap \tau / \sigma \text { and }(\rho \vee \tau) \sigma=(\rho / \sigma) \vee(\tau / \sigma)
$$

It is convenient to point out here that, for any semigroup $S$, $\Lambda(S)$ is complete ([2] p. 24) and that if $C$ is a nonvoid subset of $\Lambda(S)$ then $\mathrm{V}_{\rho \in \rho} \rho$ may be characterized as $\{(x, y) \in S \times S$ : there exist $x_{1}, \cdots, x_{n} \in S$ and $\rho_{1}, \cdots, \rho_{n+1} \in C$ (not necessarily all distinct) such that $\left.\left(x, x_{1}\right) \in \rho_{1},\left(x_{1}, x_{2}\right) \in \rho_{2}, \cdots,\left(x_{n}, y\right) \in \rho_{n+1}\right\}$. 
In order to show that a sublattice $R$ of $\Lambda(S)$ is a modular sublattice, it follows from ([1] Th. 3, p. 86) that it suffices to show that the congruences in $R$ commute; that is, that $\rho, \tau \in R$ implies that $\rho \circ \tau=\tau \circ \rho$.

We shall call a sublattice $R$ of $\Lambda(S)$ a complete sublattice if, for $C \leqq R, \mathbf{V}_{\rho \in O} \rho$ and $\bigcap_{\rho \in o} \rho$ not only exist in $\Lambda(S)$, but also belong to $R$.

Lemma 3.1. Let $S$ be a semigroup and $R=\left\{\rho_{i}: i \in I\right\}$ be a subset of $\Lambda(S)$ such that $\sigma=\bigcap_{i \in I} \rho_{i} \in R$. If $R / \sigma=\left\{\rho_{i} / \sigma: i \in I\right\}$ is a sublattice (sublattice of commuting congruences, complete sublattice) of $\Lambda(S / \sigma)$ then $R$ is a sublattice (sublattice of commuting congruences, complete sublattice) of $\Lambda(S)$.

Proof. Let $\rho_{1}, \rho_{2} \in R$. Then $\rho_{1} / \sigma \cap \rho_{2} / \sigma$ and $\rho_{1} / \sigma \vee \rho_{2} / \sigma$ belong to $R / \sigma$, as $R / \sigma$ is a sublattice of $\Lambda(S / \sigma)$. Now,

$$
\rho_{1} / \sigma \cap \rho_{2} / \sigma=\left(\rho_{1} \cap \rho_{2}\right) / \sigma \text { and } \rho_{1} / \sigma \vee \rho_{2} / \sigma=\left(\rho_{1} \vee \rho_{2}\right) / \sigma
$$

and so, by the one-to-one nature of the mapping $\rho \rightarrow \rho / \sigma$, it follows that $\rho_{1} \cap \rho_{2}$ and $\rho_{1} \vee \rho_{2}$ belong to $R$. Thus $R$ is a sublattice of $\Lambda(S)$.

Now let $R / \sigma$ be a sublattice of commuting congruences of $\Lambda(S / \sigma)$, let $\rho, \tau \in R$ and let $(a, b) \in \rho \circ \tau$. Then for some $c \in S,(a, c) \in \rho$ and $(c, b) \in \tau$. Hence $(a \sigma, c \sigma) \in \rho / \sigma$ and $(c \sigma, b \sigma) \in \tau / \sigma$. Since the elements of $R / \sigma$ commute it follows that $\rho / \sigma \circ \tau / \sigma=\tau / \sigma \circ \rho / \sigma$ and, consequently, that there exists a $d \sigma \in S / \sigma$ such that $(a \sigma, d \sigma) \in \tau / \sigma$ and $(d \sigma, b \sigma) \in \rho / \sigma$. Hence $(a, d) \in \tau$ and $(d, b) \in \rho$; that is, $(a, b) \in \tau \circ \rho$. Thus $\rho \circ \tau \subseteq \tau \circ \rho$ and likewise $\rho \circ \tau \subseteq \tau \circ \rho$. Hence $\rho \circ \tau=\tau \circ \rho$ and $R$ is a sublattice of commuting congruences of $\Lambda(S)$.

Finally, let $R / \sigma$ be a complete sublattice and let $C^{\prime} \subseteq R$. Then $\mathrm{V}_{\rho \in \sigma} \rho / \sigma$ exists and is contained in $R / \sigma$, say, $\tau / \sigma=\mathbf{V}_{\rho \in \sigma} \rho / \sigma$. Then $\rho / \sigma \leqq \tau / \sigma$, for all $\rho \in C$ and so $\rho \leqq \tau$. On the other hand, $\rho \leqq \tau^{\prime}$, for all $\rho \in C$ implies that $\rho / \sigma \cong \tau^{\prime} / \sigma$, for all $\rho \in C$, and hence that

$$
\tau / \sigma=\bigvee_{\rho \in C} \rho / \sigma \leqq \tau^{\prime} / \sigma
$$

Thus $\tau \subseteq \tau^{\prime}$ and $\mathbf{V}_{\rho \in \sigma}=\tau \in R$.

The verification that $\bigcap_{\rho \in \sigma} \rho \in R$ is even simpler. Thus $R$ is a complete sublattice of $\Lambda(S)$.

Note. It is almost immediate that if, in Lemma $3.1, R$ is a sublattice (sublattice of commuting congruences, complete sublattice) of $\Lambda(S)$ then $R / \sigma$ is a sublattice (sublattice of commuting congruences, complete sublattice) of $\Lambda(S / \sigma)$. 
Lemma 3.2. ([6] Lemma 2.2) Let $\rho$ be a congruence on a regular semigroup $S$. Then each idempotent $\rho$-class contains an idempotent of $S$.

For any semigroup $S$, let $\Sigma(\mathscr{H})=\{\rho \in \Lambda(S): \rho \subseteq \mathscr{H}\}$.

From [8] Lemmas 1 and 3, we have,

Lemma 3.3. Let $S$ be a regular semigroup. Then $\Sigma(\mathscr{H})$ is a sublattice of $\Lambda(S)$ of commuting congruences with a greatest and least element.

We call a congruence $\rho$ on a semigroup $S$ idempotent separating if each $\rho$-class contains at most one idempotent.

That any congruence $\rho$ on a semigroup such that $\rho \cong \mathscr{C}$ is idempotent separating follows from [2], Theorem 2.15, and the fact that every idempotent separating congruence on a regular semigroup is contained in $\mathscr{H}$ follows from Theorem 2.3 of [6]. Thus, for any regular semigroup $S, \Sigma(\mathscr{H})$ is the set of idempotent separating congruences on $S$.

Now any convex subset, with a largest and smallest member, of a complete lattice is clearly a complete sublattice. Hence, by Lemma 3.3, for any regular semigroup $S$, since $\Sigma(\mathscr{H})$ is clearly a convex subset of $\Lambda(S)$, it follows that $\Sigma(\mathscr{H})$ (the set of idempotent separating congruences on $S$ ) is a complete sublattice of $A(S)$.

THEOREM 3.4. Let $S$ be a regular semigroup and let

$$
\begin{aligned}
\theta & =\left\{\left(\rho_{1}, \rho_{2}\right) \in \Lambda(S) \times \Lambda(S): e \rho_{1} \cap E_{S}=e \rho_{2} \cap E_{S}, \text { for each } e \in E_{S}\right\} \\
& =\left\{\left(\rho_{1}, \rho_{2}\right) \in \Lambda(S) \times \Lambda(S): \rho_{1}\left|E_{S}=\rho_{2}\right| E_{S}\right\} .
\end{aligned}
$$

Then

(i) $\theta$ is a meet compatible equivalence on $\Lambda(S)$;

(ii) each $\theta$-class is a complete modular sublattice of $\Lambda(S)$ (with a greatest and least element).

Proof. (i) Clearly $\theta$ is an equivalence relation on $\Lambda(S)$. Let $\left(\rho_{1}, \rho_{2}\right) \in \theta$ and $\rho_{3} \in \Lambda(S)$. Then

$$
\rho_{1} \cap\left(E_{S} \times E_{S}\right)=\rho_{2} \cap\left(E_{S} \times E_{S}\right)
$$

and so

$$
\rho_{1} \cap \rho_{3} \cap\left(E_{S} \times E_{S}\right)=\rho_{2} \cap \rho_{3} \cap\left(E_{S} \times E_{S}\right)
$$

that is $\left(\rho_{1} \cap \rho_{3}, \rho_{2} \cap \rho_{3}\right) \in \theta$.

(ii) Let $A$ be a $\theta$-class, let $\sigma=\bigcap_{\tau \in A} \tau$ and let $\rho \in A$. For 
$e, f \in E_{s}$, let $(e, f) \in \rho$. Then $(e, f) \in \tau$, for all $\tau \in A$ and so $(e, f) \in \sigma$. Conversely, as $\sigma \leqq \rho,(e, f) \in \sigma$ implies that $(e, f) \in \rho$. Thus $\left.\rho\right|_{E_{S}}=$ $\left.\sigma\right|_{E_{S}}$ and $\sigma \in A$. Thus $A$ has a least member.

Now, for any $\rho \in A, \rho / \sigma$ is idempotent separating. For suppose that $f_{1}$ and $f_{2}$ are idempotents of $S / \sigma$ such that $\left(f_{1}, f_{2}\right) \in \rho / \sigma$. By Lemma 3.2, $f_{1}=e_{1} \sigma$ and $f_{2}=e_{2} \sigma$ for some idempotents $e_{1}, e_{2}$ of $S$. Thus $\left(e_{1} \sigma, e_{2} \sigma\right) \in \rho / \sigma$ and so $\left(e_{1}, e_{2}\right) \in \rho$. But $\left.\rho\right|_{E_{S}}=\left.\sigma\right|_{E_{S}}$ and so

$$
f_{1}=e_{1} \sigma=e_{2} \sigma=f_{2} .
$$

Hence $\rho / \sigma$ is idempotent separating.

On the other hand, for any congruence $\tau$ on $S / \sigma$,

$$
\tau^{\prime}=\{(a, b) \in S \times S:(a \sigma, b \sigma) \in \tau\}
$$

is a congruence on $S$. Suppose that $\tau$ is idempotent separating. If, for $e, f \in E_{S}$, we have $(e, f) \in \tau^{\prime}$, then $(e \sigma, f \sigma) \in \tau$ and so, as $\tau$ is idempotent separating, $e \sigma=f \sigma$. Thus $\left.\tau^{\prime}\right|_{E_{S}}=\left.\sigma\right|_{E_{S}}$ and $\tau^{\prime} \in A$. Now $\tau^{\prime} / \sigma=\tau$ and so $\{\rho / \sigma: \rho \in A\}$ is just the sublattice of idempotent separating congruences on $S / \sigma$. Since this, by Lemma 3.3 and the remarks following it, is a complete sublattice of $\Lambda(S / \sigma)$ of commuting congruences, we conclude from Lemma 3.1 that $A$ is a complete sublattice of $\Lambda(S)$ of commuting congruence and so a complete modular sublattice of $\Lambda(S)$.

Finally, since $A$ is a complete sublattice of $\Lambda(S), \mathbf{V}_{\rho \in A} \rho \in A$ and $A$ has a greatest member.

4. Congruences on inverse semigroups. In this and the following sections we consider inverse semigroups, for which we are able to improve on the results of the previous sections.

DeFinition 4.1. Let $S$ be an inverse semigroup and

$$
P=\left\{E_{\alpha}: \alpha \in J\right\}
$$

be a partition of $E_{S}$. Then $P$ is a normal partition of $E_{S}$ if

(i) $\alpha, \beta \in J$ implies that there exists a $\gamma \in J$ such that $E_{\alpha} E_{\beta} \subseteq E_{\gamma}$

(ii) $\alpha \in J$ and $a \in S$ implies that there exists $a \beta \in J$ such that $a E_{\alpha} a^{-1} \subseteq E_{\beta}$.

If, for an inverse semigroup $S, P=\left\{E_{\alpha}: \alpha \in J\right\}$ is a normal partition of $E_{S}$, then $E_{\alpha}$ is convex for each $\alpha \in J$. For if $e, g \in E_{\alpha}$ with $e \leqq f \leqq g$ and $f \in E_{\beta}$, then $e f=e$ implies that $E_{\alpha} E_{\beta} \subseteq E_{\alpha}$ and so $f=$ $g f \in E_{\alpha} E_{\beta} \subseteq E_{\alpha}$. Moreover, we shall denote by $\pi_{P}$ the equivalence relation on $E_{S}$ induced by $P$ and show in the following theorem that there exists a congruence $\rho$ on $S$ such that $\left.\rho\right|_{E_{S}}=\pi_{P}$. In fact, we give characterizations of the largest and smallest such congruences. 
THeOREM 4.2. Let $P=\left\{E_{\alpha}: \alpha \in J\right\}$ be a normal partition of the semilattice of idempotents of an inverse semigroup $S$. Let $\sigma=$ $\left\{(a, b) \in S \times S\right.$ : there exists an $\alpha \in J$ with $a a^{-1}, b b^{-1} \in E_{\alpha}$ and, for some $\left.e \in E_{\alpha}, e a=e b\right\}$ and $\rho=\{(a, b) \in S \times S: \alpha \in J$ implies that, for some $\left.\beta \in J, a E_{\alpha} a^{-1}, b E_{\alpha} b^{-1} \subseteq E_{\beta}\right\}$. Then $\sigma$ and $\rho$ are, respectively, the smallest and largest congruences on $S$ such that $\left.\sigma\right|_{E_{S}}=\left.\rho\right|_{E_{S}}=\pi_{P}$.

Proof. Clearly $\sigma$ is an equivalence relation. So let $(a, b) \in \sigma$ and $c \in S$, where $a a^{-1}, b b^{-1} \in E_{\alpha}$ and $e a=e b$, for some $e \in E_{\alpha}$. Now suppose that $(a c)(a c)^{-1}=a c c^{-1} a^{-1} \in E_{\gamma} \quad$ while $\quad(b c)(b c)^{-1}=b c c^{-1} b^{-1} \in E_{\delta}$. Then, since $\left(a a^{-1}\right)\left(a c c^{-1} a^{-1}\right)=a c c^{-1} a^{-1}$ and $\left(a a^{-1}\right)\left(a c c^{-1} a^{-1}\right) \in E_{\alpha} E_{\gamma}$, it follows that $E_{\alpha} E_{\gamma} \subseteq E_{\gamma}$. Likewise $E_{\alpha} E_{\delta} \subseteq E_{\delta}$. Now, eacc ${ }^{-1} a^{-1}=e a c c^{-1} a^{-1} e=$ $e b c c^{-1} b^{-1} e=e b c c^{-1} b^{-1}$ where $e a c c^{-1} a^{-1} \in E_{\alpha} E_{\gamma} \subseteq E_{\gamma}$ and $e b c c^{-1} b^{-1} \in E_{\alpha} E_{\delta} \subseteq$ $E_{\delta}$. Hence $E_{\gamma}=E_{\delta}$. Now, for any $f \in E_{\gamma}, f e \in E_{\alpha} E_{\gamma} \subseteq E_{\gamma}$ and

$$
(f e) a c=f(e a) c=f(e b) c=(f e) b c .
$$

Thus $(a c, b c) \in \sigma$.

For some $\gamma \in J, c E_{\alpha} c^{-1} \leqq E_{\gamma}$ and so $(c \alpha)(c \alpha)^{-1}=c a \alpha^{-1} c^{-1} \in c E_{\alpha} c^{-1} \subseteq$ $E_{\gamma}$ and $(c b)(c b)^{-1}=c b b^{-1} c^{-1} \in c E_{\alpha} c^{-1} \cong E_{\gamma}$. Also, if $f=c a a^{-1} e c^{-1}$ then $f \in c E_{\alpha} c^{-1} \subseteq E_{\gamma}$ and

$$
\begin{aligned}
f c a & =c a a^{-1} e c^{-1} c a=c a a^{-1} c^{-1} c e a \\
& =c a a^{-1} c^{-1} c e b=c a a^{-1} e c^{-1} c b=f c b .
\end{aligned}
$$

Thus $(c a, c b) \in \sigma$ and $\sigma$ is a congruence on $S$. Moreover, it is evident that $\left.\sigma\right|_{E_{S}}=\pi_{P}$.

Now suppose that $\tau$ is any congruence on $S$ such that

$$
\left.\tau\right|_{E_{S}}=\left.\sigma\right|_{E_{S}}=\pi_{P}
$$

and let $a, b$ be as above. Then $a a^{-1} \tau=b b^{-1} \tau=e \tau$ and so

$$
\begin{aligned}
a \tau & =\left(a a^{-1} a\right) \tau=\left(a a^{-1}\right) \tau a \tau=e \tau \alpha \tau=(e a) \tau=(e b) \tau \\
& =e \tau b \tau=b b^{-1} \tau b \tau=b \tau .
\end{aligned}
$$

Thus $\sigma \leqq \tau$ and $\sigma$ is the finest congruence on $S$ such that $\left.\sigma\right|_{E_{S}}=\pi_{P}$.

The verification that $\rho$ is the largest such congruence is similar but simpler and so we omit it.

We devote the remainder of this section to obtaining an alternative characterization of the congruences $\sigma, \rho$ of Theorem 4.2 in terms of kernel normal systems.

DEFinition 4.3 [9]. Let $S$ be an inverse semigroup. We call $\mathscr{N}$ a kernel normal system of $S$ if $\mathscr{N}$ is a collection of inverse subsemigroups of $S, \mathscr{N}^{-}=\left\{N_{\alpha}: \alpha \in J\right\}$ such that, if $E_{\alpha}=E_{N_{\alpha}}$ then 
(1) $\left\{E_{\alpha}: \alpha \in J\right\}$ is a normal partition of $E_{S}$;

(2) $a a^{-1}, b b^{-1} \in E_{\alpha}$ and $a, a b^{-1} \in N_{\alpha}$ implies that $b \in N_{\alpha}$;

(3) $a a^{-1}, b b^{-1} \in E_{\alpha}, a b^{-1} \in N_{\alpha}$ and $a E_{\beta} a^{-1} \subseteq E_{\gamma}$ implies that $a N_{\beta} b^{-1} \subseteq$ $N_{\gamma}$.

Then we have

Theorem 4.4. (Preston [9], Th. 1). Let $S$ be an inverse semigroup and let $\mathscr{N}=\left\{N_{\alpha}: \alpha \in J\right\}$ be a kernel normal system of $S$. Let $\rho_{\mathscr{r}}=\left\{(a, b) \in S \times S: a a^{-1}, b b^{-1} \in E_{\alpha}\right.$ and $a b^{-1} \in N_{\alpha}$ for some $\left.\alpha \in J\right\}$. Then $\rho_{r}$ is a congruence on $S$ and $\left\{N_{\alpha}: \alpha \in J\right\}$ is the set of idempotents in $S / \rho_{\mathscr{S}}$.

Conversely, let $\rho$ be a congruence on $S$. Then $\mathscr{N}=\left\{e_{\rho} o: e \in E_{S}\right\}$ is a kernel normal system of $S$ and $\rho=\rho_{r}$.

Thus a congruence on an inverse semigroup is uniquely determined by the congruence classes which contain the idempotents.

THEOREM 4.5. Let $S$ be an inverse semigroup and $P=\left\{E_{\alpha}: \alpha \in J\right\}$ be a normal partition of $E_{S}$. For each $\alpha \in J$, let $T_{\alpha}$ be the largest inverse subsemigroup of $S$ such that $E_{T_{\alpha}}=E_{\alpha}$, let $M_{\alpha}=\left\{x \in T_{\alpha}\right.$ : ex $=e$ for some $\left.e \in E_{\alpha}\right\}$ and let $N_{\alpha}=\left\{x \in T_{\alpha}: E_{\alpha} E_{\beta} \subseteq E_{\gamma}\right.$ implies that $x E_{\beta} x^{-1} \subseteq$ $\left.E_{\gamma}\right\}$. Then $\mathscr{C l}=\left\{M_{\alpha}: \alpha \in J\right\}$ and $\mathscr{N}=\left\{N_{\alpha}: \alpha \in J\right\}$ are kernel normal systems of $S, \rho_{\mathscr{N}}=\sigma$ and $\rho_{\mathscr{r}}=\rho$, where $\sigma$ and $\rho$ are defined as in Theorem 4.2.

Proof. For each $\alpha \in J$, let $U_{\alpha}, V_{\alpha}$ be the $\sigma$ and $\rho$-classes, respectively, of $S$ containing $E_{\alpha}$.

Clearly $M_{\alpha} \leqq U_{\alpha}$. Also, since $E_{U_{\alpha}}=E_{\alpha}$, it follows that $U_{\alpha} \leqq T_{\alpha}$. Hence for $x \in U_{\alpha}$, we have first that $x \in T_{\alpha}$. Moreover, from the definition of $\sigma$, since $x, x x^{-1} \in U_{\alpha}$, that is, $\left(x, x x^{-1}\right) \in \sigma$, we have that, for some idempotent $e \in E_{\alpha}, e x=e x x^{-1}$ and so $e x e=e x x^{-1} e=e x x^{-1} \in E_{\alpha}$. Hence $(e x e) x=e x e x=e x=e x e$ and so $x \in M_{\alpha}, M_{\alpha}=U_{\alpha}$ and $\rho_{\mathscr{M}}=\sigma$.

For $x \in N_{\alpha}$, we have $x x^{-1} \in E_{\alpha}$. Now, for any $\beta \in J, x x^{-1} E_{\beta} x x^{-1}=$ $x x^{-1} E_{\beta} \subseteq E_{\alpha} E_{\beta} \subseteq E_{\gamma}$, say. Then $x E_{\beta} x^{-1} \subseteq E_{\gamma}$, by the definition of $N_{\alpha}$, and so $\left(x, x x^{-1}\right) \in \rho$. Consequently, $x \in V_{\alpha}$. On the other hand, since $E_{V_{\alpha}}=E_{\alpha}, V_{\alpha} \subseteq T_{\alpha}$ and so $x \in V_{\alpha}$ implies that $x, x^{-1} \in T_{\alpha}$. For $\beta \in J$, suppose that $E_{\alpha} E_{\beta} \subseteq E_{\gamma}$ and let $e \in E_{\alpha}, f \in E_{\beta}$ and $g \in E_{\gamma}$. Then $e f e \in E_{\alpha} E_{\beta} E_{\alpha} \subseteq E_{\gamma}$ and so $(e \rho)(f \rho)(e \rho)=(g \rho)$. Hence, since $e \rho=x \rho=$ $x^{-1} \rho,\left(x f x^{-1}\right) \rho=(x \rho)(f \rho)\left(x^{-1} \rho\right)=g \rho$; that is, $x f x^{-1} \in E_{r}$. Since $P$ is a normal partition of $E_{S}$, it follows that $x E_{\beta} x^{-1} \subseteq E_{\gamma}$ and that $x \in N_{\alpha}$. Thus $V_{\alpha}=N_{\alpha}$ and $\rho_{\mathscr{S}}=\rho$.

5. The lattice of congruences on an inverse semigroup.

THEOREM 5.1. Let $S$ be an inverse semigroup and let 


$$
\theta=\left\{\left(\rho_{1}, \rho_{2}\right) \in \Lambda(S) \times \Lambda(S):\left.\rho_{1}\right|_{E_{S}}=\left.\rho_{2}\right|_{E_{S}}\right\} .
$$

\section{Then}

(i) $\theta$ is a congruence on $\Lambda(S)$;

(ii) each $\theta$-class is a complete modular sublattices of $\Lambda(S)$ (with a greatest and least element);

(iii) the quotient lattice $\Lambda(S) / \theta$ is complete and the natural homomorphism $\theta^{\natural}$ of $\Lambda(S)$ onto $\Lambda(S) / \theta$ is a complete lattice homomorphism.

Proof. We already know from Theorem 3.4 that $\theta$ is a meet compatible equivalence on $\Lambda(S)$. To establish that $\theta$ is a congruence it only remains to be shown that for $\left(\rho_{1}, \rho_{2}\right) \in \theta, \rho_{3} \in \Lambda(S)$ we have $\left(\rho_{1} \vee \rho_{3}, \rho_{2} \vee \rho_{3}\right) \in \theta$. Let $e \in E_{S}$ and $f \in e\left(\rho_{1} \vee \rho_{3}\right) \cap E_{S}$. Then $f \in E_{S}$ and $(e, f) \in \rho_{1} \vee \rho_{3}$. Hence there exist $x_{1}, x_{2}, \cdots, x_{k} \in S$ such that $\left(e, x_{1}\right) \in \rho_{1},\left(x_{1}, x_{2}\right) \in \rho_{3}, \cdots,\left(x_{k}, f\right) \in \rho_{3}$. Thus $\left(e, x_{1} x_{1}^{-1}\right) \in \rho_{1}\left(x_{1} x_{1}^{-1}, x_{2} x_{2}^{-1}\right) \in \rho_{3}$, $\cdots,\left(x_{k} x_{k}^{-1}, f\right) \in \rho_{3}$. But $\left(\rho_{1}, \rho_{2}\right) \in \theta$ and so $\left(e, x_{1} x_{1}^{-1}\right) \in \rho_{2},\left(x_{1} x_{1}^{-1}, x_{2} x_{2}^{-1}\right) \in \rho_{3}$, $\cdots,\left(x_{k} x_{k}^{-1}, f\right) \in \rho_{3}$. Consequently, $(e, f) \in \rho_{1} \vee \rho_{3}$ and $f \in e\left(\rho_{2} \vee \rho_{3}\right) \cap E_{S}$. Similarly $e\left(\rho_{2} \vee \rho_{3}\right) \cap E_{S} \subseteq e\left(\rho_{1} \vee \rho_{3}\right) \cap E_{S}$. Hence $\left.\rho_{1} \vee \rho_{3}\right|_{E_{S}}=\left.\rho_{2} \vee \rho_{3}\right|_{E_{S}}$ and $\left(\rho_{1} \vee \rho_{3}, \rho_{2} \vee \rho_{3}\right) \in \theta$.

Part (ii) follows immediately from Theorem 3.4.

(iii) To show that $\Lambda(S) / \theta$ is complete and the natural homomorphism $\theta$ af $\Lambda(S)$ onto $\Lambda(S) / \theta$ is complete, (i.e., $\theta$ hreserves arbitrary joins and intersections as well as pairwise joins and intersections) it is sufficient to show that $\theta$ is a complete congruence in the following sense: if, for some index set $I, \rho_{i}, \rho_{i}^{\prime} \in \Lambda(S)$ for all $i \in I$, and $\left(\rho_{i}, \rho_{i}^{\prime}\right) \in \theta$, for all $i \in I$, then

$$
\left(\bigcap_{i \in I} \rho_{i}, \bigcap_{i \in I} \rho_{i}^{\prime}\right) \in \theta
$$

and

$$
\left(\mathrm{V}_{i \in I} \rho_{i}, \mathrm{~V}_{i \in I} \rho_{i}^{\prime}\right) \in \theta
$$

However, quite minor alterations to the proofs of (i) in Theorem 3.4 and (i) in Theorem 5.1 will establish (a) and (b), respectively. Hence we have (iii).

6. Kernel normal systems. Let $S$ be an inverse semigroup and define $\theta$ on $\Lambda(S)$ as in Theorem 5.1.

Definition 6.1 [3]. Let $T$ be a semigroup, $\rho$ a congruence on $T$, and $B \cong T$. Then $[B] \rho=\{x \mid(b, x) \in \rho$ for some $b \in B\}$.

The proofs of the following two lemmas are based on the methods of Goldie [3] and Preston [9]. 
LEMMA 6.2. Let $T$ be a semigroup, $\rho_{1}, \rho_{2}$ congruences on $T$, and $B \subseteq T$. Then $\left[[B] \rho_{1}\right] \rho_{2}=\left[[B] \rho_{2}\right] \rho_{1}$ implies that $\left[[B] \rho_{1}\right] \rho_{2}=B\left(\rho_{1} \vee \rho_{2}\right)$.

Proof. (i) It is immediate from the definition that $\left[[B] \rho_{1}\right] \rho_{2} \subseteq$ $[B]\left(\rho_{1} \vee \rho_{2}\right)$.

(ii) Let $x \in[B]\left(\rho_{1} \vee \rho_{2}\right)$. Then $(b, x) \in \rho_{1} \vee \rho_{2}$ for some $b \in B$ so that there exist $x_{1}, x_{2}, \cdots, x_{k} \in T$ such that $\left(b, x_{1}\right) \in \rho_{1},\left(x_{1}, x_{2}\right) \in \rho_{2}, \cdots$, $\left(x_{k}, x\right) \in \rho_{2}$. Hence $x_{1} \in[B] \rho_{1}$ and so $x_{2} \in\left[[B] \rho_{1}\right] \rho_{2}=\left[[B] \rho_{2}\right] \rho_{1}$. Thus $x_{3} \in\left[\left[[B] \rho_{2}\right] \rho_{1}\right] \rho_{1}=\left[[B] \rho_{2}\right] \rho_{1}=\left[[B] \rho_{1}\right] \rho_{2}$. Proceeding by induction, it is easy to see that $x \in\left[[B] \rho_{1}\right] \rho_{2}$.

LEMMA 6.3. Let $\rho, \sigma \in \Lambda(S)$ be such that $(\rho, \sigma) \in \theta$ and let $\left\{N_{\alpha} \mid \alpha \in J\right\},\left\{M_{\alpha} \mid \alpha \in J\right\}$ be the kernel normal systems of $\rho$ and $\sigma$, respectively. Define $(N \vee M)_{\alpha}=\left\{k \mid k k^{-1} \in E_{\alpha}\right.$ and $k n=m$ for some $n \in N_{\alpha}$ and $\left.m \in M_{\alpha}\right\}$. Then $(N \vee M)_{\alpha}=\left[N_{\alpha}\right] \sigma=\left[M_{\alpha}\right] \rho$.

Proof. ( i ) $(N \vee M)_{\alpha} \leqq\left[N_{\alpha}\right] \sigma \cap\left[M_{\alpha}\right] \rho$. Let $k \in(N \vee M)_{\alpha}$. Then $k k^{-1} \in E_{\alpha}$ and $k n=m$ for some $n \in N_{\alpha}$ and $m \in M_{\alpha}$. Thus $k k^{-1}$, $\left(n^{-1}\right)\left(n^{-1}\right)^{-1} \in E_{\alpha}$ and $k\left(n^{-1}\right)^{-1} \in M_{\alpha}$ so that $\left(k, n^{-1}\right) \in \sigma$ and hence $k \in\left[N_{\alpha}\right] \sigma$.

Now, $k \in\left[N_{\alpha}\right] \sigma$ implies that $(k, a) \in \sigma$, for some $a \in N_{\alpha}$ and so $\left(k^{-1} k, a^{-1} a\right) \in \sigma$, where $a^{-1} a \in E_{\alpha}$. Hence $k^{-1} k \in E_{\alpha}$ and $\left(k^{-1} k, n\right) \in \rho$. Then $(k, m)=\left(k k^{-1} k, k n\right) \in \rho$ or $k \in\left[M_{\alpha}\right] \rho$.

(ii ) $\left[N_{\alpha}\right] \sigma \leqq(N \vee M)_{\alpha}$. Let $k \in\left[N_{\alpha}\right] \sigma$. Then $(k, n) \in \sigma$ for some $n$ in $N_{\alpha}$. Thus $k k^{-1}, n n^{-1} \in E_{\beta}$ and $k n^{-1} \in M_{\beta}$ for some $\beta \in J$. But $n \in N_{\alpha}$ and so $n n^{-1} \in E_{\alpha}$ so that $E_{\alpha}=E_{\beta}$. Now $k n^{-1} \in M_{\alpha}$ implies that $k \in(N \vee M)_{\alpha}$.

(iii) $[M]_{\alpha} \rho \subseteq[N \vee M]_{\alpha}$. Let $k \in\left[M_{\alpha}\right] \rho$, say $(k, m) \in \rho$ where $m \in M_{\alpha}$. Then, as $m m^{-1} \in E_{\alpha}$, we have $k k^{-1} \in E_{\alpha}$. Also, $\left(k^{-1}, m^{-1}\right) \in \rho$ and $m^{-1} m \in E_{\alpha}$ imply that $k^{-1} m \in N_{\alpha}$, say $k^{-1} m=n \in N_{\alpha}$. Then $k n=$ $k k^{-1} m \in E_{\alpha} M_{\alpha} \subseteq M_{\alpha}$ or $k \in(N \vee M)_{\alpha}$.

ThEOREM 6.4. Let $\left\{N_{\alpha} \mid \alpha \in J\right\}$ and $\left\{M_{\alpha} \mid \alpha \in J\right\}$ denote the kernel normal systems of $\rho$ and $\sigma$, respectively, where $(\rho, \sigma) \in \theta$. Let $(N \vee M)_{\alpha}=\left\{k \mid k k^{-1} \in E_{\alpha}\right.$ and $k n=m$ for some $n \in N_{\alpha}$ and $\left.m \in M_{\alpha}\right\}$ and $(N \wedge M)_{\alpha}=N_{\alpha} \cap M_{\alpha}$. Then $\left\{(N \vee M)_{\alpha} \mid \alpha \in J\right\}$ is the kernel normal system of $\rho \vee \sigma$ and $\left\{(N \wedge M)_{\alpha} \mid \alpha \in J\right\}$ is the kernel normal system of $\rho \cap \sigma$.

Proof. It is immediate that $\left\{(N \wedge M)_{\alpha} \mid \alpha \in J\right\}$ is the kernel normal system of $\rho \cap \sigma$ since for each $e \in E_{\alpha}, e(\rho \cap \sigma)=e \rho \cap e \sigma=N_{\alpha} \cap M_{\alpha}$.

If $e \in E_{\alpha}$, then $e \rho=\left[E_{\alpha}\right] \rho=N_{\alpha}$ and $e \sigma=\left[E_{\alpha}\right] \sigma=M_{\alpha}$. Since the $\theta$-classes are sublattices of $\Lambda(S), e \in E_{\alpha}$ implies that $e(\rho \vee \sigma)=$ $\left[E_{\alpha}\right](\rho \vee \sigma)$. Lemma 6.3 shows that $\left[\left[E_{\alpha}\right] \rho\right] \sigma=\left[\left[E_{\alpha}\right] \sigma\right] \rho$ and hence Lemma 6.2 implies that $\left[E_{\alpha}\right](\rho \vee \sigma)=\left[\left[E_{\alpha}\right] \rho\right] \sigma=\left[N_{\alpha}\right] \sigma=(N \vee M)_{\alpha}$. 


\section{REFERENCES}

1. G. Birkhoff, Lattice Theory, American Math. Soc. Colloquium Publications, Vol. XXV, 1964.

2. A. H. Clifford and G. B. Preston, The Algebraic Theory of Semigroups, Vol. 1, Math. Surveys of the American Math. Soc. 7 (Providence, R. I., 1961).

3. A. W. Goldie. The Jordan-Hölder Theorems for general abstract algebras, Proc, London Math. Soc. (2) 52 (1950), 107-131.

4. J. M. Howie, The maximum idempotent separating congruence on an inverse semigroup, Proc. Edinburgh Math. Soc. 14 (1964), 71-79.

5. J. M. Howie, and G. Lallement, Certain fundamental congruences on a regular semigroup, Proc. Glasgow Math. Assoc. (7). 3 (1966) 145-59.

6. G. Lallement, Congruences et equivalences de Green sur un demi-groupe regulier (to appear).

7. W. D. Munn, A class of irreducible matrix representations of an arbitrary inverse semigroup, Proc. Glasgow Math. Assoc. 5 (1961), 14-48.

8. - A certain sublattice of the lattice of congruences on a regular semigroup, Proc. Cambridge Philosophic Society 60 (1964), 385-391.

9. G. B. Preston, Inverse semigroups, J. London Math. Soc. 29 (1954), 396-403.

10. Congruences on completely o-simple semigroups, Proc. Lond. Math, Soc.

(3) 11 (1961), 557-576.

11. T. Saito, Regular elements in an ordered semigroup, Pacific J. Math. 13 (1963). 263-95.

12. V. V. Vagner, The theory of generalized groups and heaps, Mat. Sborn. (N. S.) 32 (1953), 545-632.

Received June 13, 1966 and in revised form November 8, 1966. The research for this paper was supported, in part, by NSF Grant GP-1791.

TUlane UnIVersity

The University of TeXas

Simon Fraser UNIVERSITY

The University of South Carolina 


\section{PACIFIC JOURNAL OF MATHEMATICS}

\section{EDITORS}

\section{H. ROYDEN \\ Stanford University \\ Stanford, California}

\author{
J. P. JANS \\ University of Washington \\ Seattle, Washington 98105
}

J. DugundJI

Department of Mathematics

Rice University

Houston, Texas 77001

RichaRd ARENS

University of California

Los Angeles, California 90024

\section{ASSOCIATE EDITORS}
E. F. BECKENBACH
B. H. NeumanN
F. WOLF
K. YOSIDA

\section{SUPPORTING INSTITUTIONS}

\author{
UNIVERSITY OF BRITISH COLUMBIA \\ CALIFORNIA INSTITUTE OF TECHNOLOGY \\ UNIVERSITY OF CALIFORNIA \\ MONTANA STATE UNIVERSITY \\ UNIVERSITY OF NEVADA \\ NEW MEXICO STATE UNIVERSITY \\ OREGON STATE UNIVERSITY \\ UNIVERSITY OF OREGON \\ OSAKA UNIVERSITY \\ UNIVERSITY OF SOUTHERN CALIFORNIA
}

\author{
STANFORD UNIVERSITY \\ UNIVERSITY OF TOKYO \\ UNIVERSITY OF UTAH \\ WASHINGTON STATE UNIVERSITY \\ UNIVERSITY OF WASHINGTON

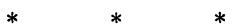 \\ AMERICAN MATHEMATICAL SOCIETY \\ CHEVRON RESEARCH CORPORATION \\ TRW SYSTEMS \\ NAVAL ORDNANCE TEST STATION
}

Mathematical papers intended for publication in the Pacific Journal of Mathematics should be typewritten (double spaced). The first paragraph or two must be capable of being used separately as a synopsis of the entire paper. It should not contain references to the bibliography. Manuscripts may be sent to any one of the four editors. All other communications to the editors should be addressed to the managing editor, Richard Arens at the University of California, Los Angeles, California 90024.

50 reprints per author of each article are furnished free of charge; additional copies may be obtained at cost in multiples of 50 .

The Pacific Journal of Mathematics is published monthly. Effective with Volume 16 the price per volume (3 numbers) is $\$ 8.00$; single issues, $\$ 3.00$. Special price for current issues to individual faculty members of supporting institutions and to individual members of the American Mathematical Society: $\$ 4.00$ per volume; single issues $\$ 1.50$. Back numbers are available.

Subscriptions, orders for back numbers, and changes of address should be sent to Pacific Journal of Mathematics, 103 Highland Boulevard, Berkeley 8, California.

Printed at Kokusai Bunken Insatsusha (International Academic Printing Co., Ltd.), 7-17, Fujimi 2-chome, Chiyoda-ku, Tokyo, Japan.

PUBLISHED BY PACIFIC JOURNAL OF MATHEMATICS, A NON-PROFIT CORPORATION

The Supporting Institutions listed above contribute to the cost of publication of this Journal, but they are not owners or publishers and have no responsibility for its content or policies. 


\section{Pacific Journal of Mathematics}

\section{Vol. 23, No. 2 \\ April, 1967}

Herbert Stanley Bear, Jr. and Bertram John Walsh, Integral kernel for

one-part function spaces .......................... 209

Mario Borelli, Some results on ampleness and divisorial schemes ....... 217

John A. Erdos, Unitary invariants for nests . ................... 229

Nathaniel Grossman, The volume of a totally-geodesic hypersurface in a pinched manifold.................................. 257

D. M. Hyman, A generalization of the Borsuk-Whitehead-Hanner theorem ............................................. 263

I. Martin (Irving) Isaacs, Finite groups with small character degrees and large prime divisors ............................. 273

I. Martin (Irving) Isaacs, Two solvability theorems ................ 281

William Lee Johnson, The characteristic function of a harmonic function in a locally Euclidean space ............................... 291

Ralph David Kopperman, Application of infinitary languages to metric spaces ............................................. 299

John Lauchlin MacDonald, Relative functor representability ............ 311

Mahendra Ganpatrao Nadkarni, A class of measures on the Bohr group.... 321

Keith Lowell Phillips, Hilbert transforms for the p-adic and p-series fields....................................... 329

Norman R. Reilly and Herman Edward Scheiblich, Congruences on regular semigroups ...

Neil William Rickert, Measures whose range is a ball ... . .

Gideon Schwarz, Variations on vector measures

Ronald Cameron Riddell, Spectral concentration for self-adjoint operators. .

Haskell Paul Rosenthal, A characterization of restrictions of

Fourier-Stieltjes transforms ................... 